Research Article

\title{
The Influence of Artificial Intelligence on Art Design in the Digital Age
}

\author{
Yan Shen ${ }^{1}{ }^{1}$ and Fang $\mathrm{Yu}^{2}$ \\ ${ }^{1}$ Design Department of Hefei University, Hefei 230601, China \\ ${ }^{2}$ Anhui Arts and Crafts Society, Hefei 230601, China \\ Correspondence should be addressed to Yan Shen; shenyan453@126.com
}

Received 12 October 2021; Revised 31 October 2021; Accepted 1 November 2021; Published 27 December 2021

Academic Editor: Tongguang $\mathrm{Ni}$

Copyright ( $) 2021$ Yan Shen and Fang Yu. This is an open access article distributed under the Creative Commons Attribution License, which permits unrestricted use, distribution, and reproduction in any medium, provided the original work is properly cited.

\begin{abstract}
With the advancement of technology represented by artificial intelligence, art creation is becoming increasingly rich, and content expression is intelligent, interactive, and data-driven, making the relationship between technology, art, and people increasingly close and bringing opportunities for the development of emerging interaction. Artificial intelligence technologies aim to perfectly replicate the human mind by enabling natural responses based on the surrounding environment, decoding emotions, and recognizing human traits within the energy range. Driven by AI technology, interactive art no longer focuses on a single audiovisual sensory experience but rather on integrated artistic expressions that are highly interactive, kinetic, and emotional, based on the study of natural human behavior and integrated senses, combined with intelligence. In this paper, we first sort out the intersection of AI technology development and interactive art expression streams on the timeline based on historical development and analyze the deconstructive relationship between the two from the macroperspective of the historical development of technology and art. First, based on the conceptual connotation, development history, technical application, and singularity outlook of AI, we identify the current characteristics and development trends of interactive art; second, based on exploring the advantages of AI technology, we propose the impact of AI on the creative thinking, creative mode, and artistic experience of interactive art and establish the paradigm of interactive art creation in the context of AI. It solves the problem that experts are unable to quickly locate the category of painters when facing different styles of unsigned digital Chinese painting images in the authenticity identification task.
\end{abstract}

\section{Introduction}

In the Internet era, digital museums, digital libraries, and some related websites of Chinese painting and art have developed. As an important art relic, digitized Chinese paintings are slowly becoming known for their advantages of easy preservation and fast retrieval. Moreover, it is simple to digitize the Chinese paintings made on Xuan paper and turn them into digital Chinese painting images [1]. Therefore, the digitization of Chinese paintings is gradually becoming a new way to protect Chinese paintings. However, experts are unable to quickly locate the category of painters in the face of unsigned digital Chinese painting images of different styles in the task of authenticity identification, which affects the efficiency of identification [2]. Therefore, the classification of Chinese painting painters, as an important branch of digital Chinese painting authenticity-assisted authentication, has gradually become a hot spot for research in terms of its classification accuracy as well as automation. On the contrary, the prevalence of Chinese painting digitization has led to the difficulty of controlling the quality of digital Chinese paintings in Chinese painting databases [3]. Moreover, in the era of big data, the sources of digitized Chinese paintings have increased, and the authenticity of their contents has gradually become a focus of attention. Traditional identification methods mainly rely on the rich experience of identification experts [4]. Due to the subjectivity of traditional identification methods and the lack of objective 
referenceable indicators, there are limitations in the efficiency and accuracy of their identification in the face of the new generation of information technology forgery methods [5]. Therefore, a digital Chinese painting authenticityassisted identification tool with a high degree of automation and accuracy is particularly necessary. At the beginning of the twentieth century, the great development of photography, communication, and other media technologies hit Western art, which was based on realism at that time and brought an unprecedented sense of crisis to art workers, and more and more artists realized that the only way to establish a new artistic status was to innovate, thus giving birth to numerous art schools [6].

Interactive art is rooted in the artistic trends of Cubism, Futurism, and Dadaism, which confronted traditional art and laid a solid foundation for the development of contemporary digital interactive art [7]. The relationship between artificial intelligence and interactive art can be traced back to the birth of the concept of "artificial intelligence" in 1956. Both interactive art and artificial intelligence technology are based on computer technology, and their inherent connection between technology and art has established the inevitability of their development and integration since their birth [8]. In the early stage of interactive art development, interactive artists customized the rules of interaction through technical research and attracted participants into the art world they created by changing the physical form, emphasizing the satisfaction of form and technical application and ignoring the experience and cognitive mode of participants. In the 1980s, computer psychology gradually developed into a cognitive discipline, artificial neural network research developed rapidly, and breakthroughs in speech recognition and machine vision technology brought artificial intelligence research into its formative years [9]. The research concept of experience formally entered the sight of artists and technology workers, and focusing on human interaction experience became the key point of interactive art expression. Therefore, as people pursue the sense of experience and entertainment in interactive art, the object of interactive art creation shifts from the study of objects to the study of human behavior, and the connection between science and art becomes closer. In the twenty-first century, with the development of deep learning technology of artificial intelligence, artificial intelligence research has entered a golden period of application, and human-computer interaction technologies such as intelligent voice interaction, machine recognition, and virtual reality based on artificial intelligence technology have advanced by leaps and bounds, which is pivotal to the development of human-centered interactive art [10]. Intelligence, nature, learning, and abiotic intelligence are new technologies and concepts carried by the development of artificial intelligence, which support the creation of contemporary digital intelligent interactive art and influence the aesthetic trends of the public and the creative thinking of artists. This paper focuses on how intelligent technology under the domination of artificial intelligence changes interactive art expression from the level of thinking, means, and experience logic and proposes a paradigm of interactive art expression under the influence of artificial intelligence technology [11].

The fusion of artistic creativity and technologically advanced productivity provides the creative impetus for the sustainable development of art and HCI technology in the future. Overall, artificial intelligence is much more than a simple technical means for art creation; it is more of a reshaping of art creation thinking and an impact on human cognition. We collect materials related to artificial intelligence, film and television creation, film and television market management, and film and television consumer experience, including books, newspapers and magazines, dissertations, theoretical works related to artificial intelligence, and film and television to provide comprehensive theoretical support for the dissertation, draw on and absorb research results from sociology, psychology, management, law, film, television, information technology, etc. The paper combines macro- and microanalysis to provide a more profound interpretation of the topic. This paper innovatively sorts out the influence of AI on interactive art expression through deconstruction and reconstruction thinking, analyzes how AI deconstructs the original model of interactive art creation in terms of technology and thinking, then analyzes the influence of AI on interactive art expression in terms of time, space, and natural logic, and establishes its application paradigm. This paper provides a new practical direction for interactive art creation. By selecting appropriate statistical methods, different external environmental factors are analyzed to understand the changes in the shape and quantity of the distribution, the relationship between variables, etc. This research method has a very obvious indirectness and it is less disturbed and easier to implement. Mainly through the analysis of statistics, statements, data, and other information published by relevant departments, we understand the development status of the film and television industry under the influence of AI, including the status of AI companies, products, and technology development related to the film and television industry and the status of AI applications in the film and television industry, and anticipate its future direction. The contributions of this work can be summarized as follows:

(1) We first sort out the intersection of AI technology development and interactive art expression streams on the timeline based on historical development and analyze the deconstructive relationship between the two from the macroperspective of the historical development of technology and art

(2) Based on exploring the advantages of AI technology, we propose the impact of AI on the creative thinking, creative mode, and artistic experience of interactive art and establish the paradigm of interactive art creation in the context of AI

(3) The proposed method can be used to quickly locate the category of painters when facing different styles of unsigned digital Chinese painting images in the authenticity identification task 


\section{Related Work}

Global research on artificial intelligence has been carried out long before, and AI cannot be called a new science. In the early development of AI, with the later development of new technologies, AI technology in recent years has made new breakthroughs in a number of areas and empowered various industries [12]. Due to the increasing frequency of AI in public life and practical applications in recent years and the gradual maturation of the technology development, the literature research is mostly focused on journal reports, and the research content involves the application of AI as a film content element and the impact of AI on the production, market management, and consumer experience in the film and television industry chain [13]. The features of Chinese paintings are used as the direct basis for intelligent classification, and the amount of information they contain directly affects the classification results. Among the features of Chinese paintings, wavelet features are used for classification [14]. The feature values that can express the characteristics of paintings are proposed as the basis of classification using the information theory. The local and global features of the paintings are used to study the authors of Chinese paintings. With further research, the artistic style of the painter becomes an important factor in the identification of the painting [15]. Features describing the painter's artistic style such as color, texture, and brushstroke also gradually became an important basis for the classification of painters. Semantic classification is achieved using the image color and shape features of the target. Color histograms, color coherence vectors, and autocorrelation texture features are used and classified into two categories according to brushwork and painting [16]. The ink color features of brush strokes and brushwork of Chinese paintings are extracted as the basis of classification. A multiscale grayscale covariance matrix method is proposed to extract texture features and complete the classification recognition of Chinese paintings [17]. Although the image content-based features such as color, texture, and brushstroke can better express the artistic style of the painting and improve the classification accuracy, the pure underlying features, again, directly lead to unsatisfactory classification results and have limitations. In addition, there are relatively few existing research works on the fusion of multidimensional features based on image content, fusing the underlying color and shape features of Chinese paintings and using the fused features as the basis of Chinese painting research [18]. The above studies fuse fewer underlying features and cannot provide a sufficient basis for analysis.

In addition to feature merit affecting intelligent classification results, the classification method and its treatment of features are also the key to intelligent classification [19]. A Monte Carlo convex packet model is used to achieve the classification of Chinese paintings. The abovementioned classification methods based on statistical models depend on the goodness of features, and the cumbersome process of tuning parameters reduces the automation of classification. They extracted wavelet features of Chinese paintings and completed the classification of Chinese paintings using support vector machines. The above machine learning-based classification methods improved the parameter tuning process, but the feature quantification was not accurate enough. With the study, it was found that deep learningbased classification methods not only provide high-level semantic representation of image features, but also the endto-end design of this class of methods improves the automation of classification. Picasso's brushstroke features are quantified using recurrent neural networks (RNNs), and painterly classification is achieved. Deep convolutional neural networks (DCNNs) are used to achieve the description of texture features of ancient paintings and the classification of ancient paintings. $\mathrm{CNN}$ is used to extract the brushstroke features in ink painting to quantify the style of ink painting and complete the classification [20]. Deep aggregation structure is used to improve the recognition ability of CNN models. The residual mechanism is proposed to increase the depth of the network by the jump connection and residual mechanism to improve the network classification performance. The feature rescaling module is proposed to emphasize the importance of different image feature channels. The convolutional block attention mechanism module (CBAM) is proposed to enhance the ability of CNN models to extract features. Therefore, it has become an important trend in the intelligent classification to introduce enhancement modules to improve the learning ability of the network based on deep learning networks.

In the 1960s and 1970s in the Western art field, research on theories related to artificial intelligence and interactive art had already begun, and many new artistic concepts were proposed, such as "artificial life art," "cyber art," and "natural intelligence" [21]. For the first time, it explores artists and their works who are working at the frontiers of scientific research and emerging technologies. The book is organized according to scientific disciplines and technological categories, discussing the in-depth relationship between art and science and technology, such as artificial intelligence and robotics, and exploring new possibilities for uncovering artists' work. It boldly predicts the next stage of artificial intelligence during the technological singularity, when human intelligences will merge with machine intelligences that greatly enrich the human brain with a faster, more accurate, larger storage, stronger memory, and efficient data-sharing capabilities. It explains the disruption of production, life, and even art by approaching singularity AI technologies, nano-life technologies, etc. It summarizes the history of the intertwining of art and technology, outlines the evolution of digital art since the 1980s, and provides a vision of future artistic expression, raising questions about audience interaction, artificial intelligence, politics, and social behaviorism through an analysis of artists' digital artworks. The third edition of this work, in 2015, expands the practice of digital art in the fields of virtual reality, augmented reality, and interactive public installations. The evolution of art forms such as interactive art-making, techno-intellectual art, and the art of dry computer and wet bio-fusion is discussed in detail. The way artificial and 
technological systems merge and the impact of future intelligence on artistic creation are explored, and the future evolution of biological self-evolution and the fate of art under the evolution of technological intelligence are looked into.

\section{Artificial Intelligence-Based Art Design Feature Extraction}

3.1. Image Features. Analysis for images is complicated by mathematical tools alone and requires finding feature values that can quantify the image information. Therefore, extracting image features and combining them with the feature learning capability of computers can improve the efficiency of image analysis. In this paper, the concepts and extraction methods of image stroke, texture, and color features are described in detail in the context of the research. Stroke features of an image are the lines that make up the content of an image, and lines are the primitives that make up the content of an image. In the image, the variation of lines can express the pose and outline of the image content. And the thickness and fiction of the lines can also convey different information. By studying the characteristics of lines on the surface of an image, a deeper meaning can be obtained. Edge detection algorithms are often used to detect line features of images. Therefore, applying edge detection algorithms to detect Chinese paintings can highlight the brushstroke features of Chinese painters. The edge detection algorithm usually relies on the edge detection operator, which is often used as the core operator for edge detection.

The detection principle is to detect the edges of an image by finding the absolute gray magnitude of the grayscale image. A series of grayscale vector information is generated when the edges of an image are detected using the reform operator. The Sobel operator usually computes two directions, horizontal and vertical, each with a convolution matrix of size $3 \times 3$, and any of the convolution matrices can be obtained by rotating another matrix by $90^{\circ}$. The expression is given in the following equation:

$$
\widetilde{E}(n)=\sum_{i=1}^{I_{k}} \sum_{P \in Q}^{n}(\lambda t)^{n+1} e^{\lambda t} \log _{n} \frac{N_{v}}{N_{n}},
$$

where $P$ is the original image and Gx and Gy are the convolution matrices in horizontal and vertical directions, respectively. The specific calculation formula is shown in equation where $f(x, y)$ is the grayscale value of the pixel point.

$$
X=\frac{X_{i}-X_{j}}{\left(\sum_{i=1}^{n}\left(X_{i}-X_{j}\right)^{2}\right)} .
$$

The calculation steps are as follows: (a) calculate the brightness difference of the image in different directions using two $3 \times 3$ matrices; (b) calculate the gradient value $G$ of the pixels in the image at Gx and Gy, and the formula is shown as follows:

$$
\begin{array}{r}
Q(x)=\frac{\sum_{i=1}^{n} p\left(X=x_{i}\right)}{\log _{2}(p(x))}, \\
Q(Y[X])=\frac{\sum_{i=1}^{n} p\left(X=x_{i}\right)}{q(x)},
\end{array}
$$

and (c) calculate the gradient direction with the following equation:

$$
\operatorname{Dis}\left(x_{i}, x_{j}\right)=\left(\sum_{i=1}^{n}\left(X_{i}-X_{j}\right)^{q}\right)^{1 / q} .
$$

In summary, the Sobel operator cannot distinguish between objects and backgrounds, and the image edges are intermittent, leading to its inaccurate results. If you want to improve the detection effect, you can increase the size of the convolution matrix, but such an operation will increase the computational effort. Usually, the image processed by the Sobel operator is not a grayscale image, which will make the image and the background not easily distinguishable. The Canny operator uses Gaussian filtering to remove image noise during edge detection, which makes the operator less susceptible to noise interference and can be applied to different scenes by setting different parameters. The Canny operator can fully reflect the strength of line edges in an image, making its detection fast and accurate.

3.2. Texture Characteristics. Texture features are surface attributes that describe an image or an image region. Regarding the extraction methods of texture features, as shown in Figure 1, they are divided into four main categories as follows:

(1) Statistical-based methods: texture features are random in the image region, but some regular characteristics of texture features can be mined using statistical methods. Statistical-based methods mainly study the grayscale distribution within the image region. Among them, the grayscale co-occurrence matrix is the most applicable, and the method uses the statistical feature values output from the grayscale co-occurrence matrix to represent the texture features of the image. The disadvantage is that it is not applicable to pixel-level texture classification tasks.

(2) Model-based methods: this class of methods considers that textures can be arranged according to some model distribution, and the relationship between texture primitives can be represented by the parameters of the model. The representative methods are mainly the random field method and fractal method. However, the disadvantages are obvious, as it does not match the actual image situation, and the texture is not adequately expressed. The fractal method mainly uses the relationship between image spatial location information and image grayscale to extract texture features of the image. The texture features extracted by this method have a 


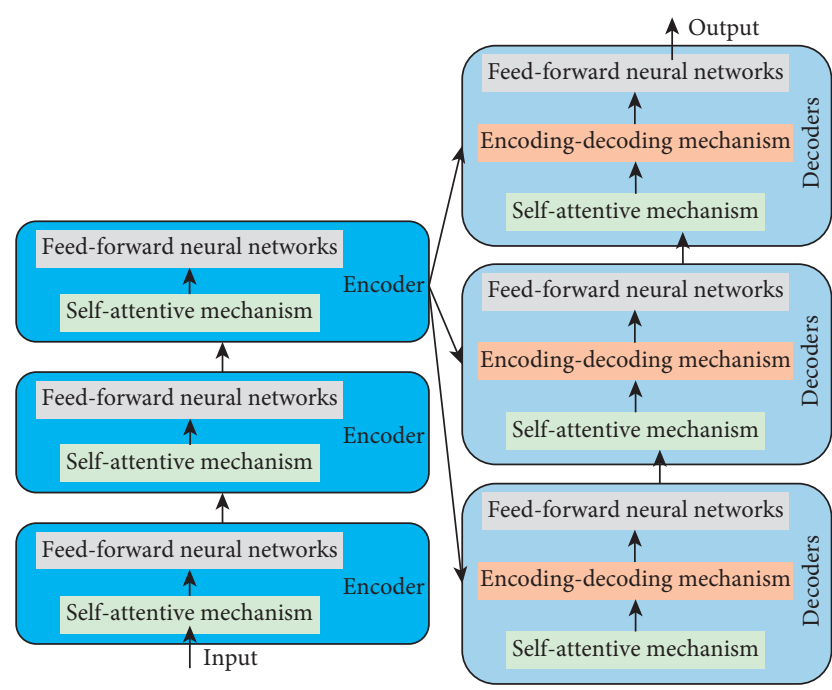

FIgURE 1: Texture feature extraction. color space and the color components. This method has an advantage in retrieval speed when facing large-scale datasets.

(3) Color moments were proposed by Stricker et al. to express the color feature information in paintings by calculating the color first-order moments, secondorder moments, and third-order moments of images, using the property that the color information is mainly concentrated in the lower-order moments.

(4) The color entropy is proposed by Zachary according to the definition of entropy mentioned by Shannon in the information theory, which combines the information entropy with the color histogram. The normalized color histogram can be set as $h$, and then the entropy of the image is expressed as

$$
E(n)=\sum_{i=1}^{k} \sum_{P \in Q}^{n}(\lambda t)^{n+1} e^{\lambda t} .
$$

characteristic that different fractal dimensions are obtained when different image regions are extracted. Therefore, applying the fractal dimension to regional texture feature extraction can easily distinguish different image regions.

(3) Spectrum-based methods: this method is mainly based on spectral characteristics and multiscale analysis of texture features. The representative method is the wavelet transform, which was first proposed in the 1990s for the texture feature extraction, and then various improved methods based on wavelet transform were applied to various tasks of image processing.

(4) Structure-based method: the basic idea of this method is to analyze the texture primitives that make up the texture features so that the regularity of the texture features can be obtained.

Color features are important visual features in image processing. Among them, red, green, and blue (RGB), hue, saturation, and lightness (HSV) are the two most commonly used color spaces. The HSV color space is more intuitive than the RGB color space in color representation and is widely used in image processing tasks. The next part of this paper focuses on the commonly used color feature extraction methods.

(1) The color histogram is the proportion of each color in the image but ignores information about the spatial distribution of colors. This method is suitable for images without segmentation tasks. The color histogram is often calculated based on the HSV color space. First, the colors in the image are divided into different regions. Then, the number of pixels of different colors in each region is counted.

(2) The color set is proposed by Smith and is similar to the color histogram. First, the HSV color space needs to be transformed and the color space quantized into a histogram. Then, an index is created between the
3.3. Artificial Intelligence Deep Learning Models. Deep learning is a new research hotspot in the field of machine learning. Deep learning mainly emphasizes two points: (1) the depth of the model and (2) the mapping of feature information learned from shallow layers to a new feature space by reasonably increasing the number of network layers, which makes the classification more accurate. The VGG deep learning model, known as Visual Geometry Group, is a deepened version of AlexNet, designed by the Department of Science and Engineering at the University of Oxford, and is often used for tasks such as image classification and face recognition. Initially, the model was designed to clarify the relationship between the depth of the network and the accuracy of large-scale classification and recognition. The VGG model is composed of a convolutional layer, a pooling layer, and a fully connected layer. The basic computation of the convolutional layer is convolutional computation. Usually, the convolution operation requires setting parameters such as step size and padding. After the convolution operation, an activation function is usually introduced. The activation function is used to increase the ability of the network to fit various kinds of data by adding nonlinear capabilities. The following activation functions are commonly used (ReLU, Sigmoid, and tanh).

In addition, after the convolution operation of the input feature map using a large number of convolution kernels, the information of the feature map needs to be filtered, and the pooling is mainly operated using the maximum and average values. The main role of the fully connected layer is to fuse the feature information, which is usually connected in descending order. The commonly used VGG models have 16 and 19 layers, respectively, as shown in Figure 2.

The performance of the VGG network is positively correlated with depth, but increasing the depth of the network will reduce the learning ability of the network and cause a lot of parameters to reduce the efficiency. GoogLeNet is designed with twenty-two layers, but with one-twelfth of the parameters of AlexNet and one-fourth of VGG. The 


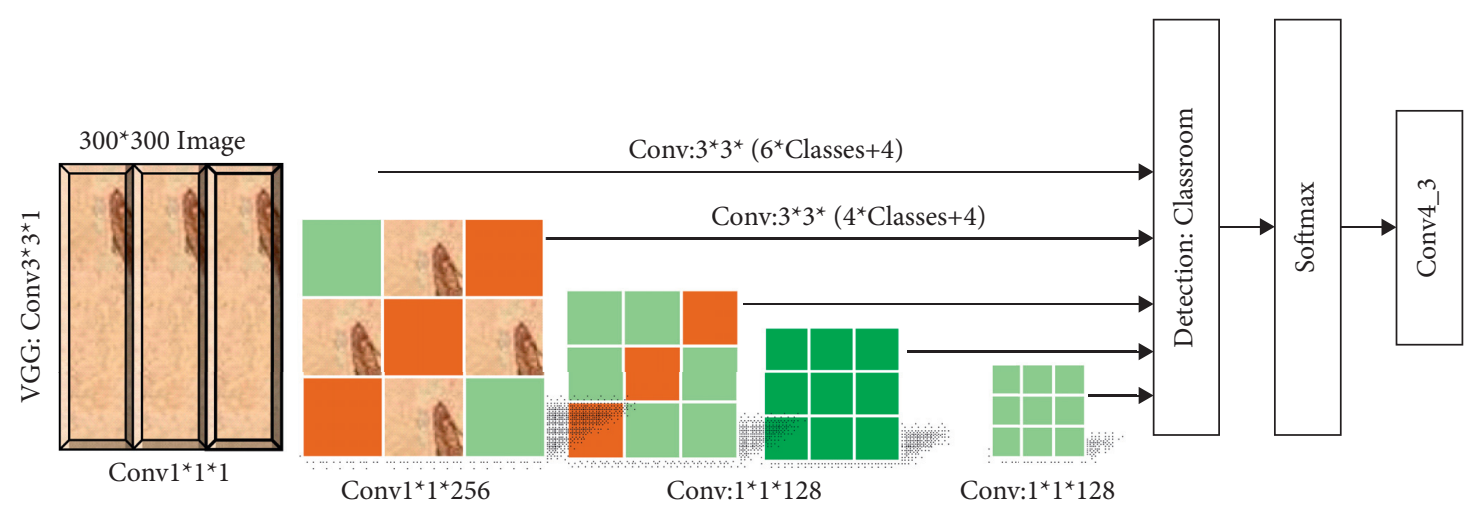

FIGURE 2: VGG-16 and VGG-19 network structures.

sparse network structure consists mainly of small convolutional kernels and $3 \times 3$ pooling layers, which can increase the network performance and ensure the efficient use of computational resources. In fact, after the network reaches a certain number of layers, the performance is negatively correlated with the number of layers, which leads to slow convergence and poor dataset performance. The residual mechanism in the residual network solves this problem by using multiple parameter layers to learn the residual representation between the input and output, that is, each learning depends on the previous learning result, preventing the performance of the network from degrading and making its learning incremental, instead of learning the mapping relationship between inputs and outputs in the ordinary order of the network structure. The advantage of the residual mechanism is that it largely solves the problem of network performance caused by increasing the number of layers. The structure of the residual mechanism is shown in Figure 3. The actual role of this layer is a nonlinear combination of features, which does not contain feature extraction and feature learning capabilities. In image processing, the number of parameters is reduced by using a small size of convolution kernels.

\section{Analysis of Experimental Results}

The experiments in this paper are based on the PyTorch deep learning framework for training and testing, with a NVIDIA GTX 1060 GPU, 8 GB of video memory, and Cuda version 10.1. The programming language is Python 3.6. Figure 4 shows the main parameters of the network training, and these parameters are used as the basic settings for the comparison experiments. To verify the performance of the proposed network classification in the context of Chinese painting classification, a five-fold cross-validation method is used, where the entire dataset is first divided into five disjoint subsets, and then four of them are randomly used as the training set and one as the test.

In the image block rejection process, the artistic target of the painting occupies a certain proportion of the whole frame, and there are blank and meaningless image blocks in addition to the artistic target because the blank image blocks and the image blocks containing a small number of artistic targets can provide less information and have the characteristic of low threshold. Therefore, the optimal contrast threshold is selected among [20, 60, 100, 140, 180, 220] using the grid search method. Among the random field methods, Markov random field models are very common, and they are used to establish a connection between textures and twodimensional image fields. As can be seen from Figure 5, when the threshold value is 20 , the presence of image blocks with invalid information leads to a low accuracy rate. As the contrast threshold value increases, the accuracy also increases. This is because blank image blocks and image blocks containing little invalid information are eliminated and image blocks containing valid information are retained. The accuracy is highest at the threshold value of 180 . When the threshold value is 220 , the accuracy starts to decrease because the blocks containing valid information are incorrectly rejected, which affects the network classification results. Therefore, the threshold is set to 180 , and the specific effect of the image contrast-based image block rejection method on different subjects and images of different frame formats. From the results, it is shown that the method is good at removing blank and meaningless image blocks from paintings and retaining their artistic target details.

From Figure 5, it can be seen that the check-all rate and check-accuracy rate of color features are low among the four painters, which is because the color features are extracted with a single color variety in the paintings, and the color richness of the dataset is poor, making it difficult to achieve good classification results. This phenomenon is more obvious in Xu Beihong's paintings. As can be seen from the figure, the check-all rate and check-accuracy rate of $\mathrm{Xu}$ Beihong's paintings are obviously low, and the color features in Xu Beihong's paintings are not sufficiently characterized. $\mathrm{Xu}$ Beihong's paintings are mainly ink and wash, that is, black and white, lacking rich color expressions, which leads to lower check-all and check-accuracy rates. Thus, it can be seen that color features have a certain characterization ability, but the low color richness of traditional ink paintings and the little feature information available lead to the limitations of color features in the classification. Compared with the color features, the texture features have better characterization ability, and the texture features have better performance in two indexes, the check-all rate and the 


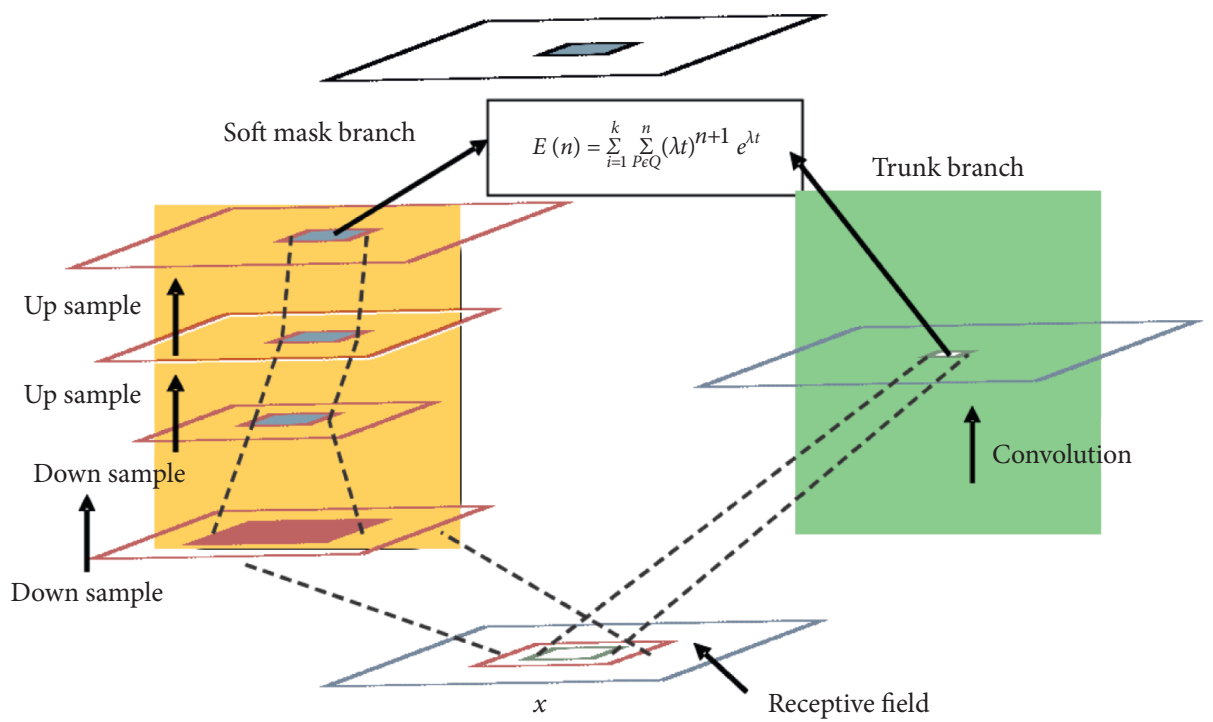

Figure 3: Residual mechanism structure diagram.

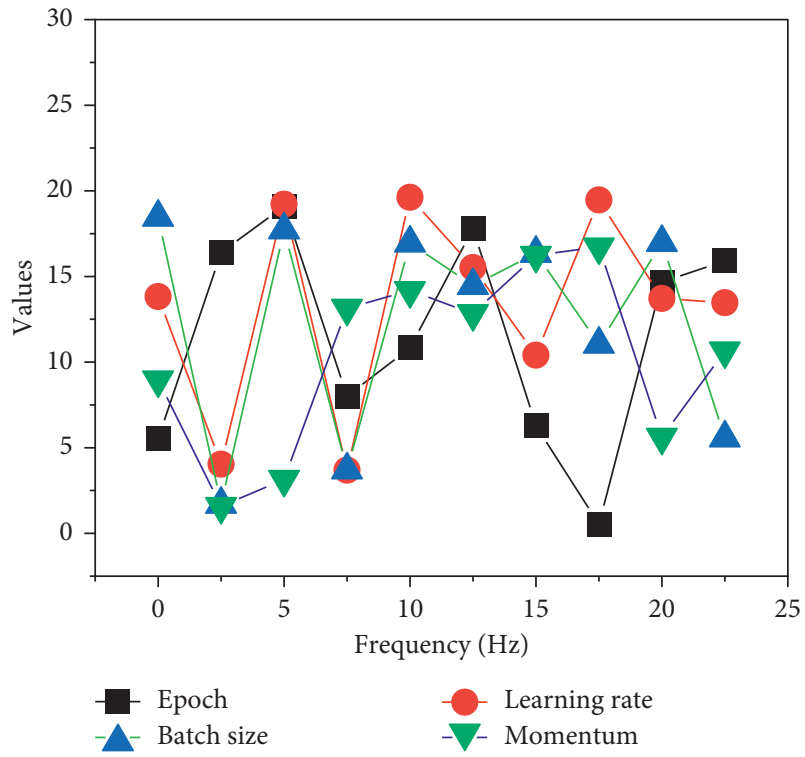

FIGURE 4: Network parameter data.

check-accuracy rate. The texture feature map processed by the local binary mode algorithm removes the interference of the color feature of the painting itself, and the rotation invariance also reduces the influence of the different forms of the painting. In the four-painter classification experiments, the brushstroke features have better performance in two technical indexes, namely, the detection rate and the accuracy rate, compared with the color and texture features. It indicates that the brushstroke feature map processed by the edge detection algorithm can quantify the painter's artistic style by detecting the edge contours of the painter's brushwork. Finally, after fusing the three features, the checkall rate and check-accuracy rate of the four painters are significantly improved. It indicates that the fused features can make up for the deficiency of the color features' characterization ability; they have a deep semantic expression of

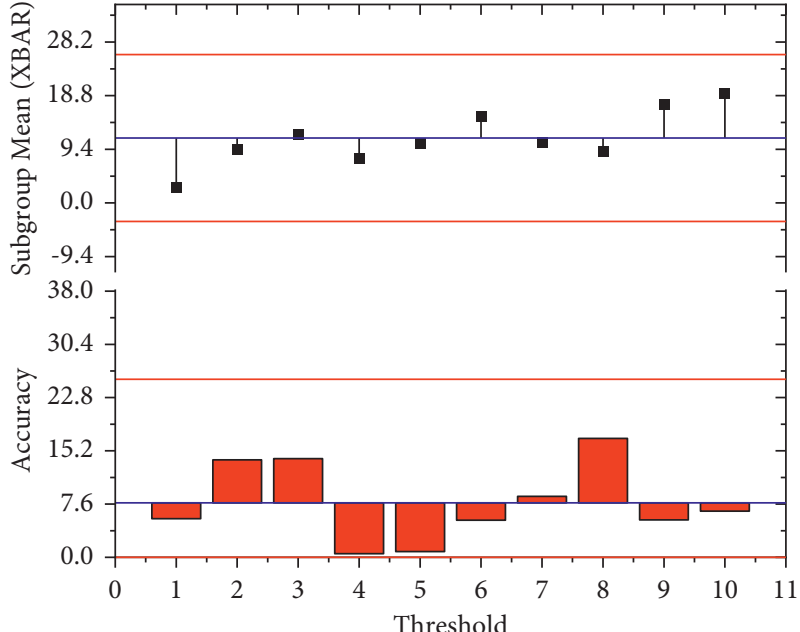

Figure 5: Different contrast thresholds.

the texture features; they retain the stroke features that can express the painter's artistic style. In summary, the fusion features are more suitable as the basis for the network classification. Furthermore, we discuss the effectiveness of the proposed network, and first argue whether the convolutional attention mechanism enhances the performance of the network. In the deep convolutional network, the commonly used attention mechanism modules are the SE module and CBAM module, and the experiments are mainly focused on these two modules to verify. The specific experimental results are shown in Figure 6.

From the figure below, it can be seen that the CBAM module slightly outperforms the SE module in terms of the average accuracy metric. It indicates that the SE module emphasizes the relationship between network channels but ignores the spatial information of the feature map. The natural logic expressed by intelligent interactive art follows the knowledge given to human beings by the natural human system. The social nature of human beings determines the 


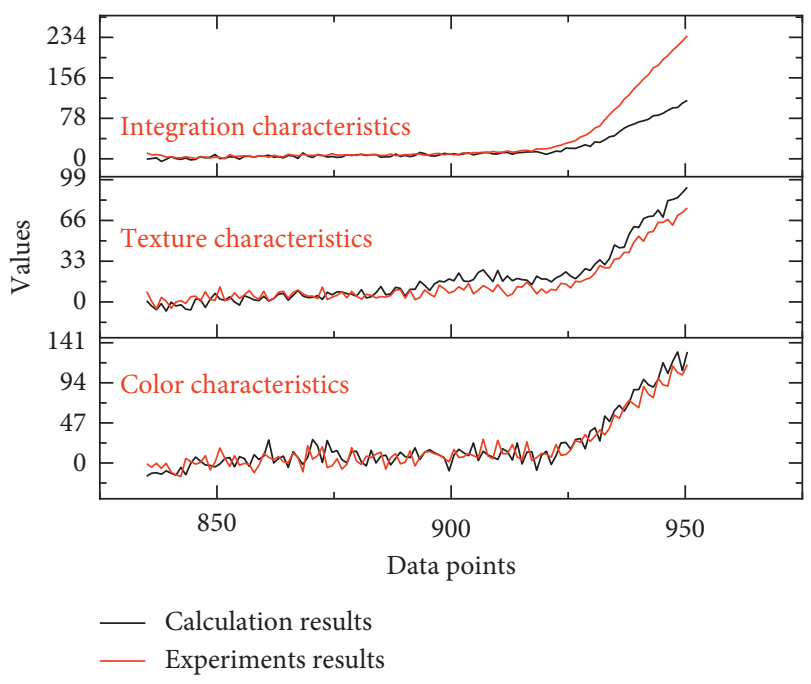

Figure 6: Experimental results of feature comparison.

transmission and dissemination of this experience so that we have today an extremely rich human cognitive experience and spiritual culture. The CBAM module compensates this deficiency by emphasizing the relationship between the feature map channels and learning the spatial information of the feature map through the spatial attention mechanism module. Compared with the base network, the CBAM module improves the feature extraction capability of the network and significantly enhances the performance of the network. Therefore, we incorporate this module into the network. After incorporating the CBAM module, the network has significantly improved in the average accuracy index, but during the experiment, we found that the performance of the network is directly affected when the number of CBAM modules incorporated into the network varies. Then, this paper compares the relationship between the number of CBAM modules and the performance of the network. The number of CBAM modules is incremented by the number of integrated modules in the experiment. As seen in Figure 7, when no CBAM modules are added to the network, the performance is similar to that of the ResNet-50 network. As the number of CBAM modules increases, the performance of the network gradually improves. When CBAM modules are incorporated in each residual unit, it not only increases the feature extraction capability of the network but also improves the classification performance of the network.

Usually, deep learning networks use ReLU as the activation function to increase the network's ability to be nonlinear. We experimentally compare the Mish activation function with the ReLU activation function. From the experimental results, we can see that there is a slight improvement in the average accuracy metric after using the Mish activation function. It indicates that the Mish activation function can propagate information better in the network and increase the nonlinear capability of the network, which makes its performance of extracting features enhanced. To verify the advantages of the proposed network in this paper, we compared the network with three current mainstream

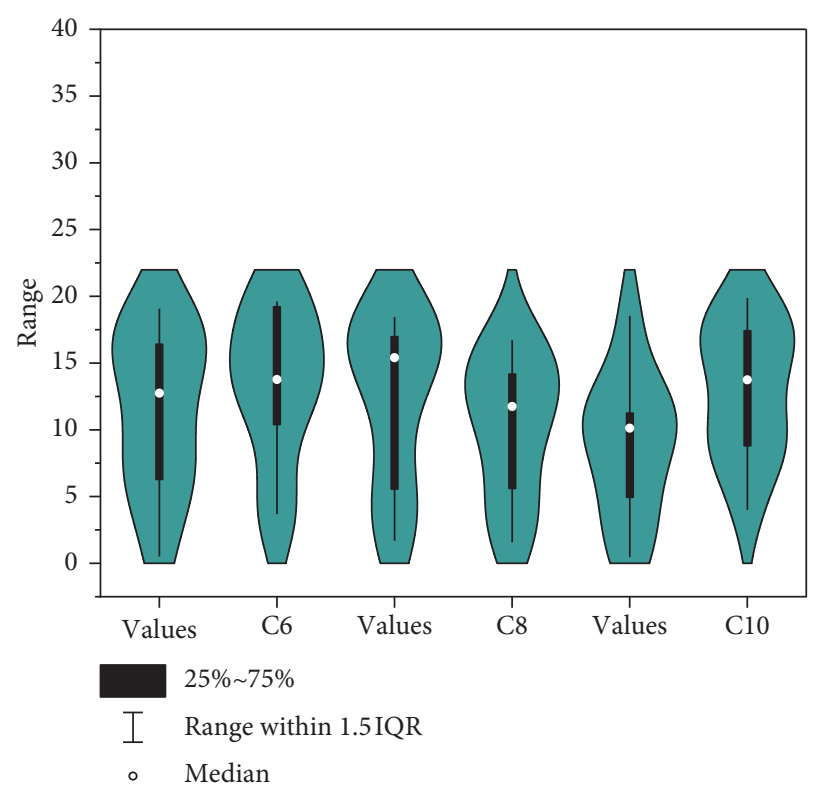

FIgURE 7: Experimentation with the number of CBAM modules.

classification networks and measured them with the average accuracy metric. The results are shown in Figure 8. Compared with the VGG-16, GoogLeNet, and ResNet-50 networks, the proposed network outperforms the other mainstream networks in terms of the average accuracy metric. It indicates that the multibranch attention mechanism network proposed in this paper has advantages in feature extraction and fusion classification and is suitable for tasks such as intelligent classification of national paintings.

The fact that man comes from nature determines that man can never isolate himself from nature. When exploring the reasons for the rise of modern science in the West, he pointed out that the essence of the Renaissance was a revival of science and technology and proposed that Mr. Art and the son of Science belongs to the same essence of nature. Nature is the totality of all objective realities in the universe and the origin of all things. What distinguishes intelligent interactive art from other forms of interactive art is its adherence to natural logic and the intelligent technology put into practice. Human cognitive behavior consists of two parts; the first part is the action system that expresses the basic characteristics of human beings, which is generated in the environmental needs and collective behavioral examples, such as waving, nodding, and other human-specific physical actions; the second part is the cognitive system transmitted by language or words, which is the feedback mechanism generated by environmental needs and cognitive evolution, and this feedback mechanism, through the processing of external information, produces knowledge that combines speech, text, emotion, physical objects, and specific internal environmental information. As the aesthetic subject of intelligent interactive art, the human being completes the emotional transmission of intelligent interactive art. In this process, artificial intelligence provides visitors with human basic movements and cognitive recognition conditions under the human feedback mechanism, completes the 


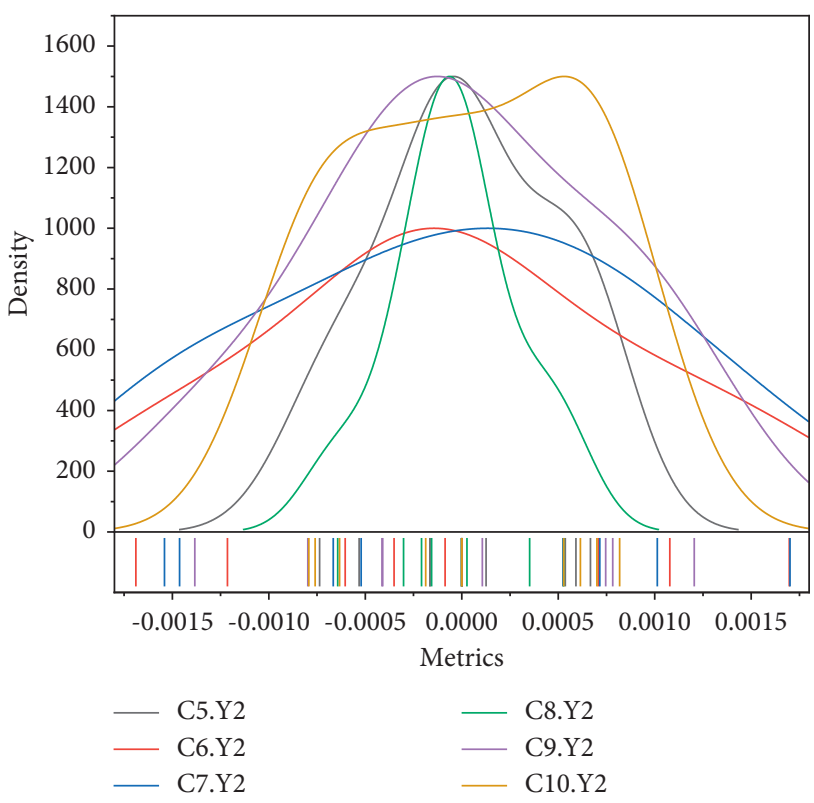

Figure 8: Average accuracy metrics measurement.

smooth interaction with the work, realizes the natural integration of subject, object, and surrounding environment, and then forms the real immersion experience and the final emotional transmission.

\section{Conclusion}

In this paper, an intelligent classification algorithm for Chinese painting painters based on a multibranch attention mechanism network is proposed from multifeature fusion and deep semantic representation of features. Firstly, the algorithm uses contrast statistical features to eliminate digital Chinese painting image blocks with little information; secondly, the features are pregenerated for digital Chinese paintings using the style characteristics of Chinese paintings; then, an intelligent classification network based on multibranch attention mechanism is designed to complete the fusion and classification of Chinese painting features.

Based on the analysis of the innate advantages of AI technology, this study proposes how AI can change the original paradigm of interactive art expression application from the level of creative thinking, creation mode, and art experience so as to establish an intelligent interactive art creation model in the context of AI. Based on the study of interactive art expression form under the influence of $\mathrm{AI}$ technology, it proposes that AI changes the original concept of art aesthetics from space, time, and natural logic. Based on the study of interactive art expressions under the influence of AI technology, we propose the changes of AI on the original concept of art aesthetics in terms of space, time, and natural logic and analyze its aesthetic characteristics of deboundaryization, immersion, and emotionality. This paper focuses on the deconstruction and reconstruction of interactive art expression by artificial intelligence. This paper proposes a digital Chinese painting authenticity-assisted authentication method based on twin networks. Firstly, we use adversarial generative networks to learn the characteristics of painters' paintings and generate corresponding painters' forgeries; secondly, we propose a deep learningbased digital Chinese painting authenticity-assisted identification network for the generated forgeries; then, we visualize the characteristic regions of painters' images; finally, we develop an authenticity-assisted identification system based on the web, which can produce more accurate-assisted identification results and provide experts with an objective basis for experts. Through experimental analysis, it is proved that the proposed method has good sensitivity to digital forgeries and is scalable.

In the future, the brushstroke feature map processed by the edge detection algorithm can quantify the painter's artistic style by detecting the edge contours of the painter's brushwork. In addition, after a large amount of data collection, we may encounter the problem of big data analysis. At this time, we will use some lightweight optimization models to solve the problem of big data analysis. In the near future, we will further use artificial intelligence methods to learn and simulate the artistry of painters so as to realize the ability of automatic painting of artificial intelligence.

\section{Data Availability}

The data used to support the findings of this study are included within the article.

\section{Conflicts of Interest}

The authors declare no conflicts of interest.

\section{Acknowledgments}

This study was supported by Phased achievements of the major national philosophy and Social Sciences (Art) project "Research on design aesthetics" (approval no. 19zd23).

\section{References}

[1] J. Banfield, "Challenge in artistic flow experiences: an interdisciplinary intervention," Qualitative Research in Psychology, vol. 18, no. 1, pp. 104-125, 2021.

[2] R. Bonatti, W. Wang, C. Ho et al., "Autonomous aerial cinematography in unstructured environments with learned artistic decision-making," Journal of Field Robotics, vol. 37, no. 4, pp. 606-641, 2020.

[3] V. Y. Borisov, E. M. Galyamova, and Z. A. Pershina, "Features of professional training of primary school teachers in the field of artistic and graphic activities in the context of digital education," Science and School, no. 1, pp. 104-115, 2021.

[4] C. Cahill, D. Alberto Quijada Cerecer, A. R. Reyna Rivarola, J. Hernández Zamudio, and L. Alvarez Gutiérrez, "Caution, we have power': resisting the 'school-to-sweatshop pipeline' through participatory artistic praxes and critical care," Gender and Education, vol. 31, no. 5, pp. 576-589, 2019.

[5] R. A. Campbell, E. J. Bradshaw, N. Ball, A. Hunter, and W. Spratford, "Effects of digital filtering on peak acceleration and force measurements for artistic gymnastics skills," Journal of Sports Sciences, vol. 38, no. 16, pp. 1859-1868, 2020. 
[6] K. Moreno Gata and E. Echeverría Valiente, “The use of digital tools for the preservation of architectural, artistic and cultural heritage, through three-dimensional scanning and digital manufacturing," The International Archives of the Photogrammetry, Remote Sensing and Spatial Information Sciences, vol. XLII-2/W9, pp. 501-506, 2019.

[7] E. Gourgou, A. R. Willis, S. Giunti et al., "A journey to 'tame a small metazoan organism', $\ddagger$ seen through the artistic eyes of C. elegans researchers," Journal of Neurogenetics, vol. 34, no. 3-4, pp. 549-560, 2020.

[8] A. C. Hu, B. J. Sun, and W. K. Y. Ng, "Is there a role for artistic training in surgery? A multi-institutional assessment of general surgeons and plastic surgeons," Journal of Plastic, Reconstructive \& Aesthetic Surgery, vol. 74, no. 4, pp. 890-930, 2021.

[9] A. Abbas, M. Choi, J. Seo, S. H. Cha, and H. Li, "Effectiveness of immersive virtual reality-based communication for construction projects," Ksce Journal of Civil Engineering, vol. 23, no. 12, pp. 4972-4983, 2019.

[10] C. Ackermann, M. Beggiato, S. Schubert, and J. F. Krems, "An experimental study to investigate design and assessment criteria: what is important for communication between pedestrians and automated vehicles?" Applied Ergonomics, vol. 75, pp. 272-282, 2019.

[11] S. A. Baker and M. J. Walsh, "'Good morning fitfam': top posts, hashtags and gender display on instagram," New Media \& Society, vol. 20, no. 12, pp. 4553-4570, 2018.

[12] M. Dhieb, M. Al-Amri, and A. Jamil, "The digital urban atlas of jeddah: some raised issues and semiological principles," Current Urban Studies, vol. 7, no. 2, pp. 265-287, 2019.

[13] C. Forceville and S. Paling, "The metaphorical representation of depression in short, wordless animation films," Visual Communication, vol. 20, no. 1, pp. 100-120, 2021.

[14] R. Hamaguchi, S. Nematollahi, and D. J. Minter, "Picture of a pandemic: visual aids in the COVID-19 crisis," Journal of Public Health, vol. 42, no. 3, pp. 483-485, 2020.

[15] Z. Jafri, N. Ahmad, M. Sawai, N. Sultan, and A. Bhardwaj, "Digital Smile Design-An innovative tool in aesthetic dentistry," Journal of Oral Biology and Craniofacial Research, vol. 10, no. 2, pp. 194-198, 2020.

[16] S. Jahnavi and C. Nandini, "Novel multifold secured system by combining multimodal mask steganography and naive based random visual cryptography system for digital communication," Journal of Computational and Theoretical Nanoscience, vol. 17, no. 12, pp. 5279-5295, 2020.

[17] C. Kearns, "Is drawing a valuable skill in surgical practice? 100 surgeons weigh in," Journal of Visual Communication in Medicine, vol. 42, no. 1, pp. 4-14, 2019.

[18] F. Kurasawa, "On humanitarian virality: kony 2012, or, the rise and fall of a pictorial artifact in the digital age," Visual Communication, vol. 18, no. 3, pp. 399-423, 2019.

[19] M.-J. Lee and J.-H. Pae, "Photo-fake conditions of digital landscape representation," Visual Communication, vol. 17, no. 1, pp. 3-23, 2018.

[20] A. Lehmuskallio, J. Häkkinen, and J. Seppänen, "Photorealistic computer-generated images are difficult to distinguish from digital photographs: a case study with professional photographers and photo-editors," Visual Communication, vol. 18, no. 4, pp. 427-451, 2019.

[21] S. McDonough and E. Colucci, "People of immigrant and refugee background sharing experiences of mental health recovery: reflections and recommendations on using digital storytelling," Visual Communication, vol. 20, no. 1 , pp. 134-156, 2021. 